\title{
Reduced Resting-State Functional Connectivity in Current and Recovered Restrictive Anorexia Nervosa
}

\author{
Jessica Clare Scaife ${ }^{1 *}$, Lauren Rose Godier ${ }^{1}$, Nicola Filippini ${ }^{1,2}$, Catherine J. Harmer ${ }^{1}$ and \\ Rebecca J. Park'
}

'Department of Psychiatry, University of Oxford and Oxford Health NHS Foundation Trust, Warneford Hospital, Oxford, UK, ${ }^{2}$ FMRIB Centre, University of Oxford, Oxford, UK

OPEN ACCESS

Edited by:

Paul Croarkin,

Mayo Clinic Minnesota, USA

Reviewed by:

Farana Farzan,

University of Toronto, Canada

Cara Bohon,

Stanford University, USA

Stephan Zipfel,

University of Tübingen, Germany

*Correspondence:

Jessica Clare Scaife

jessica.scaife@psych.ox.ac.uk

Specialty section:

This article was submitted to Neuroimaging and Stimulation,

a section of the journal

Frontiers in Psychiatry

Received: 19 October 2016 Accepted: 08 February 2017

Published: 28 March 2017

Citation:

Scaife JC, Godier LR, Filippini N,

Harmer CJ and Park RJ (2017)

Reduced Resting-State

Functional Connectivity in Current

and Recovered Restrictive

Anorexia Nervosa.

Front. Psychiatry 8:30.

doi: 10.3389/fpsyt.2017.00030
Functional connectivity studies based on resting-state functional magnetic resonance imaging (rs-fMRI) have shown alterations in brain networks associated with self-referential processing, cognitive control, and somatosensory processing in anorexia nervosa (AN). This study aimed to further investigate the functional connectivity of resting-state networks (RSNs) in homogenous subsamples of individuals with restrictive AN (current and recovered) and the relationship this has with core eating disorder psychopathology. rs-fMRl scans were obtained from 12 female individuals with restrictive AN, 14 females recovered from restrictive AN, and 16 female healthy controls. Independent components analysis revealed a set of functionally relevant RSNs, previously reported in the literature. Dual regression analysis showed decreased temporal coherence within the lateral visual and auditory RSNs in individuals with current AN and those recovered from AN compared to healthy individuals. This decreased connectivity was also found in regions associated with somatosensory processing, and is consistent with reduced interoceptive awareness and body image perception, characteristic of AN. Widespread gray matter (GM) reductions were also found in both the AN groups, and differences in functional connectivity were no longer significant when GM maps were added as a covariate in the dual regression analysis. This raises the possibility that deficits in somatosensory and interoceptive processing observed in AN may be in part underpinned or exacerbated by GM reductions.

Keywords: anorexia nervosa, fMRI, resting-state, neural connectivity, gray matter

\section{INTRODUCTION}

Anorexia nervosa (AN) is a psychiatric disorder in which the relentless and compulsive pursuit of self-starvation leads to severe malnourishment. Recovery rates are low and around a quarter of those affected go on to develop a chronic course of the disorder (1). It has the highest mortality rate of any psychiatric disorder (2) and is one of the most debilitating diseases in young women (3). AN remains one of the most challenging psychiatric disorders to treat, particularly in adults (4). There are few evidence-based psychological treatments (5) and very little evidence that there are pharmacological agents which give benefit (6-8). In the quest to develop novel interventions, there has been increasing interest in disease mechanisms $(9,10)$, in particular, neurobiological factors and processes underlying AN $(11,12)$. A neurobiological account of AN based on dysfunctional ventral limbic and dorsal executive circuitry has been proposed (11), but relatively few studies have directly investigated aberrancies in the neural circuitry of individuals with current AN. 
This study used resting-state functional magnetic resonance imaging (rs-fMRI), in which the temporal synchronicity of spatially separate brain regions is measured. This cross-brain synchronicity is thought to reflect functional networks integral to information processing (13). fMRI blood oxygenation leveldependent (BOLD) signal is measured in individuals at rest, while performing no particular task. BOLD signals are then correlated in time (14). Resting-state networks (RSNs) are comprised of regions which show strong temporal coherence and are thus believed to be functionally connected (15).

There are a number of different approaches to analyzing resting-state data. In the present study, we used a whole-brain independent components analysis (ICA), a data-driven approach which does not require a priori regions of interest and looks at the patterns of temporal synchronicity between brain regions which emerge while the brain is at rest $(16,17)$. Along with a few studies which have also used whole-brain ICA, the AN literature also contains RSN studies using other approaches, such as seed-based analysis, network-based ICA, and graph analysis. Differences in both methodology and population studied have limited direct comparisons between studies [see the review by Gaudio and colleagues (18)].

In the first published study of rs-fMRI in individuals recovered from AN, our group found increased connectivity within the default mode network (DMN) between the precuneus and dorsolateral prefrontal cortex (DLPFC) using whole-brain ICA (19). The DMN incorporates parts of the prefrontal cortex including the DLPFC, the cingulate cortex, and the precuneus and is more active at rest than during active tasks (20). It is believed to be involved in stimulus-independent thought and self-reflection. The DLPFC and anterior cingulate are also involved in cognitive control and may be responsible for excessive control over appetite and food reward in $\operatorname{AN}(11,21,22)$. Increased connectivity of the insula and the DMN in those with a current diagnosis of anorexia nervosa has also been reported (23), which may normalize in recovery (24). However, some RSN changes do persist into recovery, such as changes in the connectivity of regions in the frontoparietal network (24) and our finding of increased connectivity in the DMN (19).

Differences in networks implicated in cognitive control have also been observed, including increased connectivity of the DLPFC and ventromedial PFC, regions associated with cognitive inflexibility (25). Reduced connectivity of the inferior frontal gyrus (IFG) within the ventral attention network has also been reported (26). The IFG is part of the cognitive control system of the brain which governs response inhibition (27). Furthermore, alterations in connectivity between the thalamus and the prefrontal cortex have also been seen in $\mathrm{AN}$, which may play a role in the cognitive dysfunction seen in this group (28). In a study using whole-brain ICA to examine RSNs in a population of adolescents with AN, decreased functional connectivity between the executive network and the anterior cingulate was reported, which might contribute to impaired cognitive flexibility in relation to the control of appetite and body image in AN (29).

Consistent with the changes in body image perception characteristic of AN, altered connectivity patterns associated with disturbed body image have also been seen. Favaro and colleagues found decreased connectivity in the ventral-visual network in both acute and recovered AN groups and increased connectivity in the somatosensory network in the acute AN group. These results were correlated with visuospatial abilities and were suggested to reflect the inability to integrate visual and somatosensory perceptual information. This inability might sustain body image disturbance in AN (30). Phillipou and colleagues also found decreased functional connectivity between sensorimotor and visual networks in AN (31). A number of studies have also reported changes in the insula in $\mathrm{AN}$ : increased functional connectivity between the insula and the cerebellum in the cerebellar-parietal network (32) and reduced connectivity with the thalamus has been reported $(33,34)$. These findings may help to explain the impairments in body awareness and body perception in AN.

This study is a novel application of the exploratory whole-brain ICA approach in a group which includes a sample of restrictive currently ill individuals and a group who have recovered from AN. Only two previous studies have used whole-brain ICA to study resting-state functional connectivity in $\mathrm{AN}$, and these studies were methodologically different to this study. In our previous study (19), the resting-state scan followed a disorder-related task, which may have impacted on rumination. Furthermore, we previously only included a recovered sample. In the study by Gaudio and colleagues (29), only adolescents with only a short history of AN were included. Functional differences between this group and healthy controls (HCs) are likely to be less extreme than in those who have experienced chronic AN.

In this study, we sought to address a number of limitations in our previous study (19). While our previous approach of studying individuals recovered from AN (21) has the advantage of removing the confounding effect of acute starvation on neural activity (11), including an acutely ill sample in the current study may allow us to begin separating impairments associated with a history of AN from those associated with the starvation state. We also focus exclusively on understanding restrictive AN, reducing heterogeneity of the AN sample. Restrictive and binge/purge subtypes of $\mathrm{AN}$ are associated with cognitive and neurobiological differences (35), and by isolating a purely restrictive sample, we aimed to lower within-group variation, thereby allowing between-group differences to be more easily detected. In contrast to our previous study, the resting-state scan for this study was carried out prior to any task components, to avoid the potential effects of task in the activity of RSNs.

In this study, we predicted that with this more homogenous sample and in the absence of any bias from a previous symptom-provocation task, RSNs associated with somatosensory and interoceptive processes will show changes in connectivity, particularly in the current $\mathrm{AN}$ group, consistent with altered body image perception and interoception reported in the illness (10).

\section{PARTICIPANTS AND METHODS}

\section{Participants}

Forty-two participants were recruited for three experimental groups: 12 female individuals with anorexia nervosa (AN; 
restrictive subtype), 14 recovered (AN-R) females, and 16 female HCs (Table 1). General exclusion criteria for all subjects were left-handedness and magnetic resonance imaging (MRI) contraindications. General inclusion criteria for the HC group were a normal body mass index (BMI) (18.5-25), maintained for the 12 months prior to the study and Eating Disorder Examination Questionnaire (EDE-Q) scores within 1 SD of the global mean scores for young women, no evidence of current or past psychiatric disorder, no history of neurological or significant medical illness, and no first degree relative with a current or past eating disorder diagnosis. Inclusion criteria for the AN-R included a previous DSM-IV diagnosis of AN, absence of significant eating disorder pathology for at least 12 months prior to the study (e.g., restricting, excessive exercise, bingeing, purging), maintenance of a BMI within normal range (18.5-25) for the 12 months prior to the study, and EDE-Q scores within $1 \mathrm{SD}$ of the EDE-Q global mean scores for young women. Inclusion criteria for the AN group included a current DSM-IV (Diagnostic and Statistical Manual of Mental Disorders, fourth Edition) diagnosis of anorexia nervosa, confirmed using the eating disorder examination interview and being currently underweight in BMI $(<17.5$, calculated during the screening session).

In the AN group, primary diagnosis was AN; however, there were some comorbidities: two also fulfilled criteria for comorbid Major Depressive Disorder (MDD), one currently suffered from comorbid Generalized Anxiety Disorder (GAD), and five presented with both MDD and GAD. In the AN group, five participants were on SSRI/SNRI antidepressant drugs, and three participants were also taking atypical antipsychotic drugs. In the AN-R group, three participants were taking SSRI/SNRI antidepressant drugs.

\section{Measures}

The Structured Clinical Interview for DSM-V Axis I Disorders (36) was used to screen for DSM-V Axis-I disorders. ED psychopathology was measured using the Eating Disorder
Examination (37), which refers to the previous 28 days. During screening, participants completed the Yale-Brown-Cornell Eating Disorder Scale Self-Report Questionnaire (YBC-EDSSRQ) (38), which indexes eating-related preoccupations and or rituals.

Depressive symptoms were measured using the BDI-II (39). Anxiety symptoms were measured using the State-Trait Anxiety Inventory (40) on the day of screening, which is a 40 -item selfreport instrument that captures both state and trait anxiety. Verbal IQ was measured using the National Adult Reading Test (NART) (41), with non-native English speakers excluded from group means.

On the day of the scan, participants were asked not to eat for $4 \mathrm{hrs}$ prior to the fMRI scan and to drink only water or caloriefree drinks. Participants completed Visual Analog Scales for the dimensions "how do you see yourself" (very thin-very fat) and "fear of weight gain" (0-100 mm, not at all-extremely) to assess feelings about body image immediately prior to the scan.

\section{Neuroimaging Protocol}

Scanning was performed at the University of Oxford, Centre for Clinical Magnetic Resonance Research (OCMR) using a 3-T Siemens Trio scanner with a 32-channel head-coil. The neuroimaging protocol comprised functional and structural sequences as follows.

\section{Resting-State Functional Magnetic Resonance Imaging}

Whole-brain functional imaging was performed using a gradientechoEPIsequence $\left(\mathrm{TR}=2,000 \mathrm{~ms}, \mathrm{TE}=28 \mathrm{~ms}\right.$, flip angle $=89^{\circ}$, field of view $=224 \mathrm{~mm}$, voxel dimension $=3 \mathrm{~mm} \times 3 \mathrm{~mm} \times 3 \mathrm{~mm}$, acquisition time $=6 \min 4 \mathrm{~s}$ ). For the resting-state scan, subjects were instructed to lie in dimmed light with their eyes open, think of nothing in particular, and not to fall asleep.

A functional scan involving a food-pictures task was performed following the resting-state scan, prior to the structural MRI, data are not reported in this paper, see Ref. (42).

TABLE 1 | Demographics, mood questionnaire and eating disorder scores in the three groups $(M \pm S D$, one-way ANOVA $p$-scores).

\begin{tabular}{|c|c|c|c|c|}
\hline Sociodemographic data & $\begin{array}{l}\text { Healthy Controls (HCs) } \\
\qquad(N=16)\end{array}$ & $\begin{array}{l}\text { Recovered (AN-R) } \\
(N=14)\end{array}$ & $\begin{array}{l}\text { Current AN (AN) } \\
\qquad(N=12)\end{array}$ & p-Score \\
\hline Age & $24.3 \pm 5.7$ & $27 \pm 6.5$ & $29.4 \pm 6.0$ & 0.092 \\
\hline National Adult Reading Test & $109.6 \pm 6.8$ & $114.6 \pm 6.8$ & $114.7 \pm 8.3$ & 0.159 \\
\hline Age of onset of anorexia nervosa (AN) (years) & N/A & $16.5 \pm 2.1$ & $20.1 \pm 5.9$ & 0.04 \\
\hline Duration of AN (years) & N/A & $5.8 \pm 4.2$ & $10.3 \pm 5.2$ & 0.02 \\
\hline EDE & $0.2 \pm 0.2$ & $0.7 \pm 0.6$ & $2.8 \pm 1.6$ & $<0.001^{*}$ \\
\hline STAI-State & $26.9 \pm 6.5$ & $34.4 \pm 5.4$ & $51.2 \pm 15.5$ & $<0.001^{*}$ \\
\hline STAI-Trait & $32.9 \pm 9.7$ & $45.2 \pm 10.6$ & $61.7 \pm 14.0$ & $<0.001^{\star \star}$ \\
\hline $\mathrm{BDI}$ & $3.3 \pm 4.5$ & $6.4 \pm 6.4$ & $30.3 \pm 18.7$ & $<0.001^{*}$ \\
\hline YBC-EDS- SRQ current & $\mathrm{N} / \mathrm{A}$ & $3.8 \pm 3.9$ & $15.2 \pm 7.7$ & $<0.001$ \\
\hline YBC-EDS-SRQ past & $\mathrm{N} / \mathrm{A}$ & $23.8 \pm 5.3$ & $23.3 \pm 5.6$ & 0.82 \\
\hline
\end{tabular}

Post hoc differences between AN and HCs indicated by *, between both AN and HC and AN-R and HC indicated by **. Where two groups were compared, $p$-Score is from independent samples $t$ tests.

BMI, body mass index; NART, National Adult Reading Test; STAl, State-Trait Anxiety Inventory; BDI, Beck Depression Inventory; EDE, eating disorder examination; VAS, Visual Analog Scales; YBC-EDS-SRQ, Yale-Brown-Cornell Eating Disorder Scale Self-Report Questionnaire. 


\section{Structural MRI}

Structural MRI was acquired at the end of the two functional MRI sequences. 3D high-resolution T1-weighted MR images were acquired using a magnetization-prepared rapid gradient-echo sequence $\left(\mathrm{TR}=2,040 \mathrm{~ms}, \mathrm{TE}=4.7 \mathrm{~ms}\right.$, flip angle $=8^{\circ}$, field of view $=192 \mathrm{~mm}$, voxel dimension $=1 \mathrm{~mm}$ isotropic, acquisition time $=6 \mathrm{~min})$.

\section{Analysis of RSNs}

Resting-state functional magnetic resonance imaging analysis of resting-state data was carried out using Multivariate Exploratory Linear Optimized Decomposition into Independent Components (MELODIC, part of FSL: http://www.fmrib.ox.ac. $\mathrm{uk} /$ fslmelodic/) (43). Individual pre-processing consisted of motion correction, brain extraction, spatial smoothing using a Gaussian kernel of full width at half maximum (FWHM) $5 \mathrm{~mm}$ and high pass temporal filtering with a cut-off of $150 \mathrm{~s}$ $(0.007 \mathrm{~Hz})$. rs-fMRI volumes were registered to the individual's structural scan and standard space images using Oxford Centre for Functional MRI of the Brain's (FMRIB) Non-linear Image Registration Tool (FNIRT). This data analysis was performed in three stages:

First, the pre-processed functional data containing 180 timepoints for each subject were temporally concatenated across subjects in order to create a single $4 \mathrm{D}$ dataset. Then, the (groupwise) concatenated multiple rs-fMRI datasets were decomposed using a group ICA to identify large-scale patterns of functional connectivity in the population of subjects.

Second, dual regression was carried out on the data, which allowed voxel-wise comparisons of resting functional connectivity to be made between subjects (44). Within each subject's rs-fMRI dataset, subject-specific temporal dynamics and spatial maps that are associated with each group ICA map were identified. This is a two-step process, which involves first the use of the full set of group-ICA spatial maps in a linear model fit (spatial regression) against the separate rs-fMRI datasets, resulting in matrices describing temporal dynamics for each component for each subject. Second, these subject-specific time-course matrices are used to perform temporal regression (linear model fit) against the associated rs-fMRI dataset to estimate subject-specific spatial maps. In the final step of the group ICA, the different component maps are collected across subjects into single 4D files (one per original ICA map, with the fourth dimension being subject ID). At this stage, independent components of interest were identified, and subjected to further analysis.

Third, a voxel-wise general linear model (GLM) based analysis was used to assess group differences in each spatial map using permutation-based non-parametric testing (5,000 permutations) (45) with cluster-based thresholding using Threshold-Free Cluster Enhancement (TFCE) and a family-wise-error corrected cluster significance threshold of $p<0.05$ applied to the suprathreshold clusters. This results in spatial maps characterizing the betweensubject/group differences. The GLM comparison included the groups of interest comparison (current $\mathrm{AN}$ vs controls, recovered $\mathrm{AN}$ vs controls, current and recovered AN vs controls). Each of the contrasts of interest was tested for group averages and difference between groups.

Non-parametric tests were used to safeguard against the possibility that the between-subjects effects were non-Gaussian, and because such non-parametric inference has greater robustness against spatial non-stationarity than commonly used parametric methods (46). Because previous evidence suggested that acquisition of rs-fMRI data after a task may potentially confound results (47), we acquired our data prior to the food image task, which is not reported in this paper.

\section{Analysis of Structural MRI}

Whole-brain analysis was carried out using a voxel-based morphometry-style analysis (FSL-VBM) (48) using default settings as described at www.fmrib.ox.ac.uk/fsl/fslvbm/. In brief, brain extraction and tissue-type segmentation were performed and resulting gray matter (GM) partial volume images were aligned to standard space using first linear (FLIRT) and then non-linear (FNIRT) registration tools. The resulting images were averaged, modulated, and smoothed with an isotropic Gaussian kernel of $5 \mathrm{~mm}$ FWHM to create a study-specific template, and the GM images were re-registered to this, including modulation by the warp field Jacobian. In the figures, the result maps are reported in accordance with neurological convention (right is right).

Voxel-wise GLM analysis was carried out using permutation testing in a similar fashion as described for the rs-fMRI analysis. Permutation-based non-parametric testing (5,000 permutations) (45) with cluster-based thresholding using TFCE and a familywise error (FWE)-corrected cluster significance threshold of $p<0.05$ applied to the suprathreshold clusters.

For each subject, GM, white matter (WM), and cerebrospinal fluid (CSF) volumes were derived and computed as a percentage of total brain volume. This was compared between groups and GM volume in the AN group was correlated with BMI, age, lowest BMI, duration of disease and past YBC-EDS-SRQ scores.

Statistical analyses of non-imaging variables were carried out using SPSS software (SPSS, Inc., Chicago IL, USA) version 22.0. Threshold for statistical significance was set to $p<0.05$.

\section{Covariates}

Structural images were used as additional covariates on a voxel by-voxel basis to interrogate rs-fMRI data. GM images of each subject were extracted using FMRIB's Automated Segmentation Tool (http://fsl.fmrib.ox.ac.uk/fsl/fslwiki/FAST/), registered to standard space, smoothed to match the intrinsic smoothness of the rs-fMRI data, voxel-wise demeaned across all subjects in both groups together and added as a confounding regressor (nuisance) to the GLM design matrix used to analyze rs-fMRI data. Adding GM maps reduces variance in the data due to potentially confounding anatomical differences between subjects (49). Due to the (non-significant) trend toward group differences in age $(p=0.092)$, a subsequent analysis also included age as a covariate. The results did not survive the inclusion of age as a covariate. When examining group differences in SPSS, age was added as a post hoc covariate. 


\section{RESULTS}

\section{Demographic and Psychological Characteristics}

Table 1 shows the demographic and psychological characteristics of the groups. Duration of illness was significantly greater in the AN than the AN-R group: $t(1,24)=-2.45, p=0.02$. Age of onset was significantly later in the AN than the AN-R group: $t(1,24)=-2.17, p=0.04$. Lowest BMI, past YBC-EDS-SRQ scores, and NART did not differ between groups $(p>0.05)$. The AN group reported significantly higher state anxiety and depression (BDI) levels than HC group. The AN group also reported higher trait anxiety than the AN-R group. The AN-R group also had higher trait anxiety than HC but state anxiety and depression were not significantly different. There was a significant group effect on the measure "how afraid are you of gaining weight". Both AN groups were significantly more afraid of gaining weight than $\mathrm{HC}(F=17.0, p<0.001)$, the AN group were also more afraid of gaining weight than the AN-R group $(p<0.05)$.

\section{Resting-State Functional Connectivity}

Independent component analysis (ICA) defined 33 independent components. Of these, nine components were identified as RSNs (covering the majority of GM) and were evaluated further (Figure 1, shows nine RSNs). The other components reflected distinct artifacts resulting from head motion and physiological or scanner noise. The RSNs of interest included: DMN, salience, medial visual, lateral visual, auditory, motor, executive control, and frontoparietal (right and left). These networks
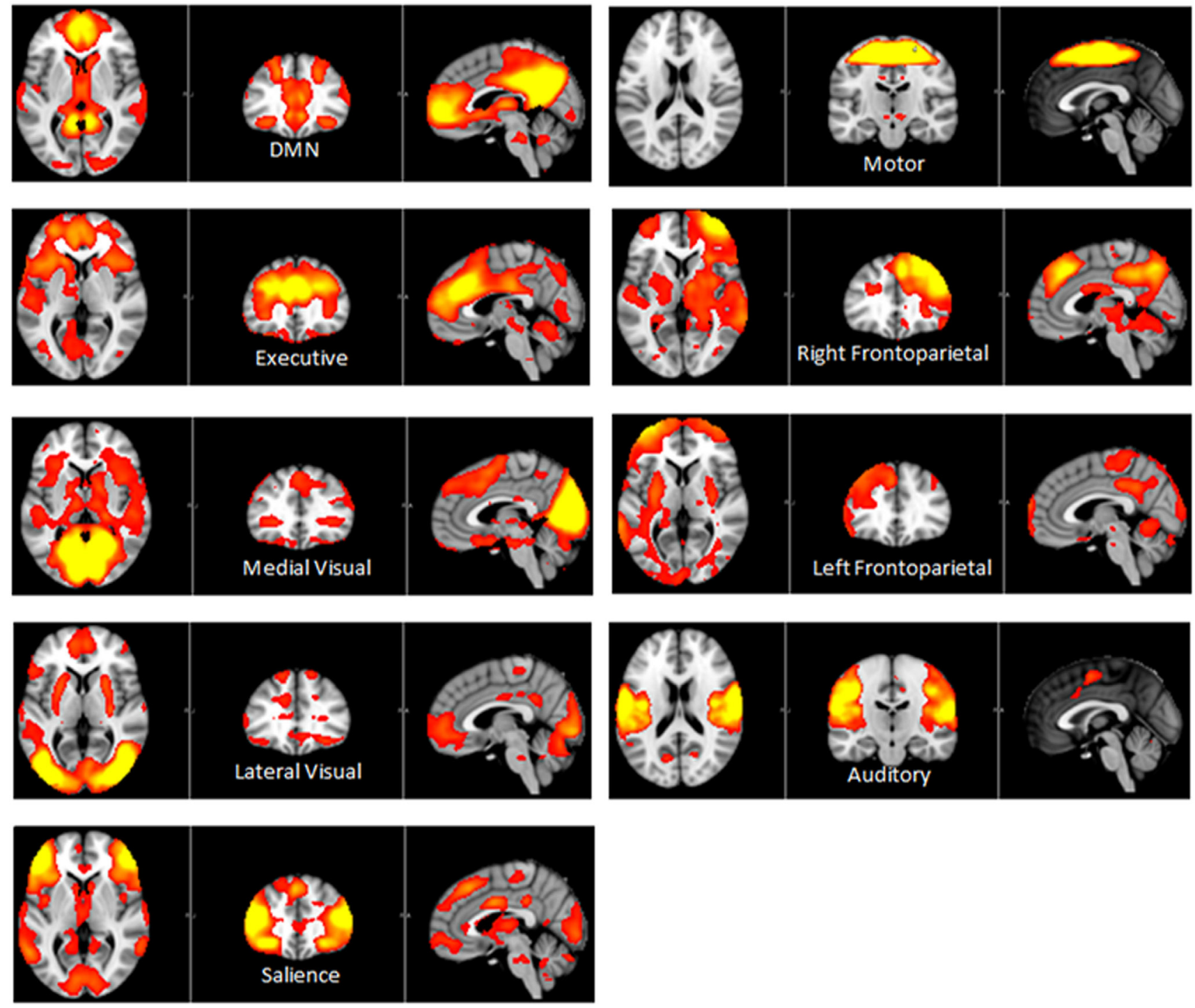

FIGURE 1 | Axial, coronal and sagittal slices for the main RSNs detected, overlaid onto the standard MNI brain. 
corresponded to RSNs described previously with high stability over time (43).

The results of the GLM found significant between-group differences in the voxel-wise spatial distribution of the functional connectivity maps in the lateral visual and auditory networks. Significantly decreased temporal correlation (coherence) was observed in the contrast: HC greater than AN and AN-R within the auditory network in clusters in the following regions: the right insula extending into the central opercular cortex, the left opercular cortex, right Heschl's gyrus, the right precentral gyrus extending into the inferior frontal and postcentral gyri, the left precentral and postcentral gyri (peak $64,-12,18, t=4.44$, voxel size 819; see Figures 2 and 3). Significantly decreased temporal correlation (coherence) was observed in AN and AN-R groups compared to $\mathrm{HC}$ group within the lateral visual network in the temporal fusiform cortex extending into the temporal-occipital fusiform cortex and lingual gyrus (peak 38, $-36,-14, t=4.04$, voxel size 176; see Figures 2 and 3). A box plot of the extracted values for all three groups, is shown in Figure 4.

Total scores on the YBC-EDS-SRQ (past) did not significantly correlate with either RSN dual regression output $p>0.05$.

\section{Structural MRI}

The data for global CSF, WM, and GM volumes are reported in Table 2. There was no significant between-group difference in $\mathrm{WM}$ volume $(F[2,39]=0.8, p=0.45)$. There was a significant between-group difference in CSF volume $(F[2,39]=6.83$, $p=0.004)$, driven by an increase in CSF volume in the AN group compared with AN-R $(p=0.032)$ and $\mathrm{HC}(p=0.004)$.

There was a significant between-group difference in GM volume $(F[2,39]=4.868, p=0.013)$ driven by reduction in GM in the AN group compared with HC $(p=0.012)$. There was no significant difference in global GM between the AN group and the AN-R group $(p=0.093)$. In the results of the GLM, the contrast $\mathrm{HC}>\mathrm{AN}$ revealed a widespread reduction in GM in the brains of individuals with $\mathrm{AN}$, with significant clusters in the left precentral gyrus, left middle frontal gyrus, bilateral inferior frontal gyrus, right lateral prefrontal cortex, dorsal anterior cingulate/ juxtapostional lobule, left planum polare-insular cortex, bilateral thalamus, bilateral hippocampus, bilateral putamen, left angular gyrus/supramarginal gyrus, and superior parietal lobule $p<0.05$ (Figure 5). There were no brain regions in which the AN group had increased GM density volume compared with AN-R and HC groups.

Global GM volume did not significantly differ between the AN-R and HC groups; however, in the GLM (contrast $\mathrm{HC}>\mathrm{AN}-\mathrm{R}$ ), there was a significant cluster in the right supramarginal gyrus/superior parietal lobule, $p<0.05$ (Figure 5). The overlap in regions of GM reduction in $\mathrm{AN}$ and $\mathrm{AN}-\mathrm{R}$ groups is seen in Figure 6.

\section{GM Maps as Covariates}

To determine whether BOLD differences at rest between the groups were influenced by the significant GM reduction in
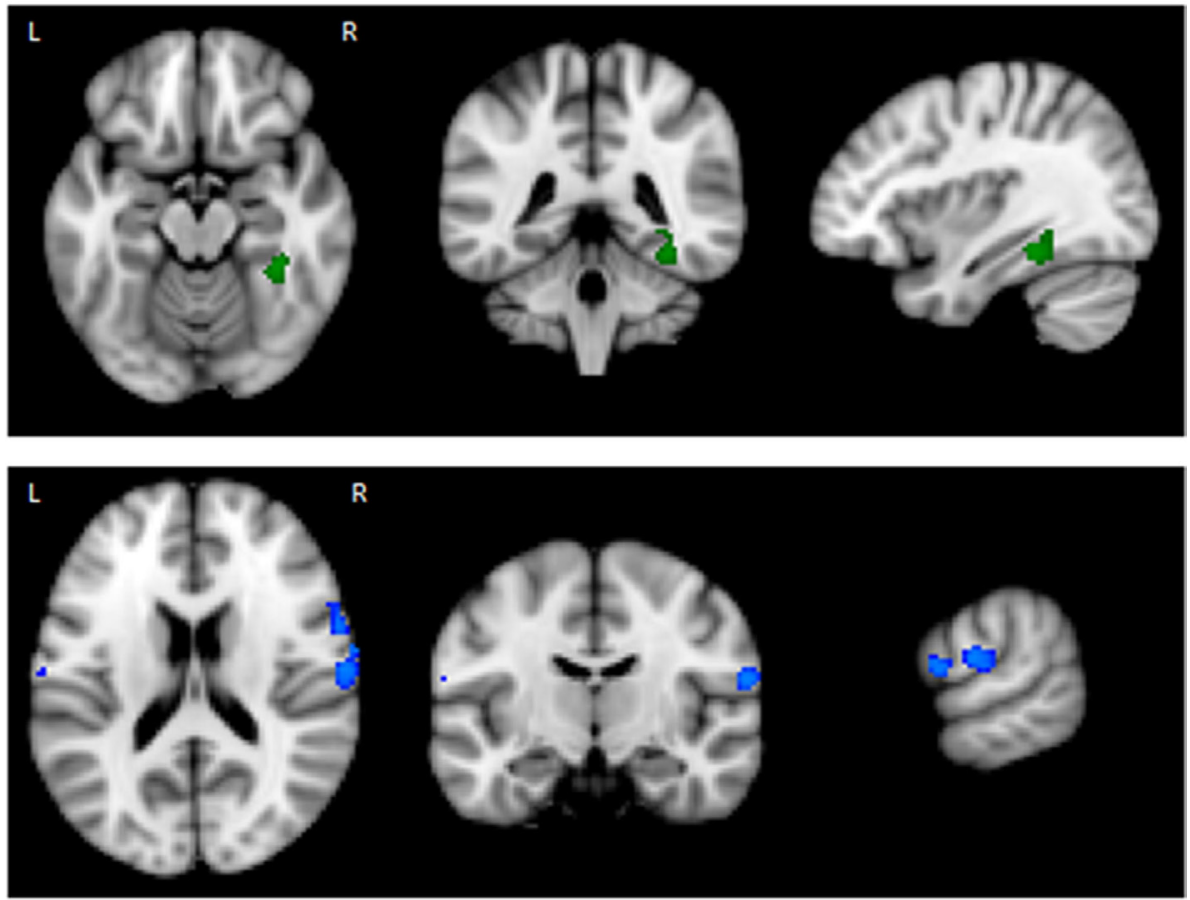

FIGURE 2 | Top: Regions of decreased connectivity with the Lateral Visual Network, the temporal fusiform cortex is shown. Bottom: Regions of decreased connectivity within the Auditory Network, the precentral and postcentral gyri are shown. 

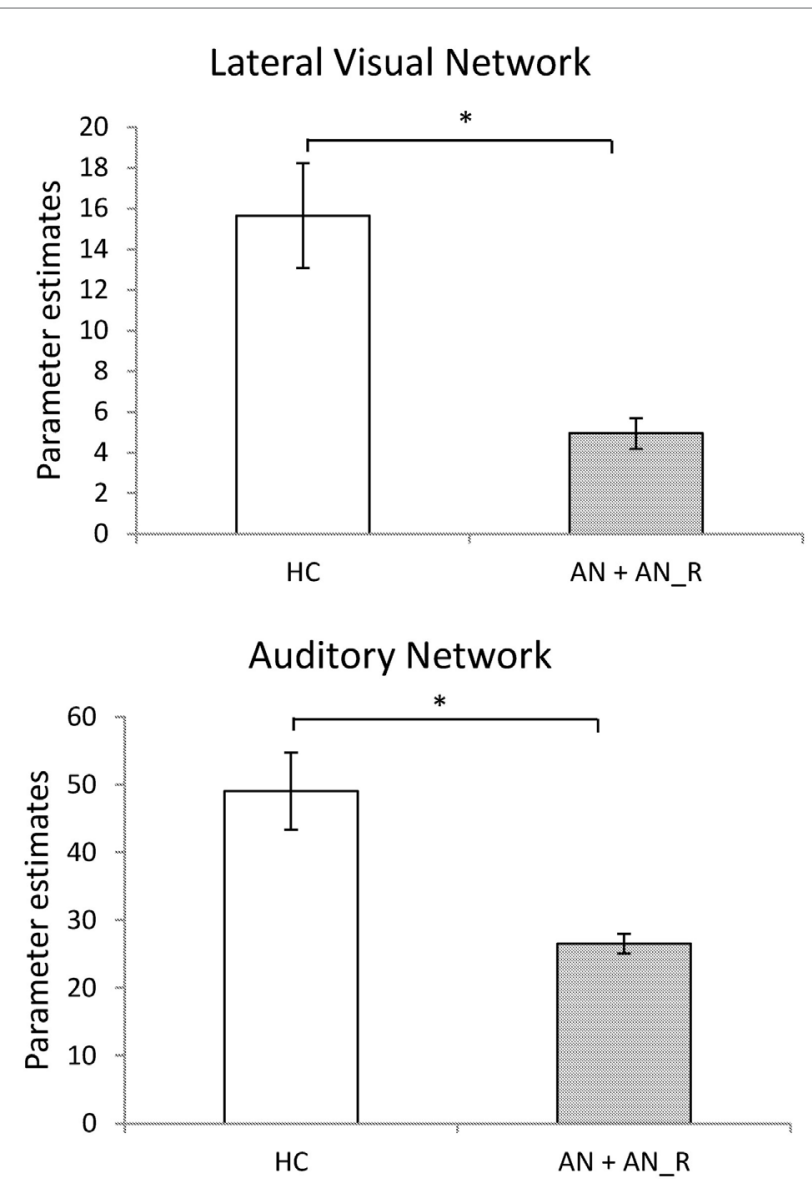

FIGURE 3 | Significantly reduced functional connectivity in the pooled $\mathrm{AN}$ and $\mathrm{AN}-\mathrm{R}$ groups compared to the $\mathrm{HC}$ group in the Lateral Visual Network (Top) and Auditory Network (Bottom).

the AN and AN-R groups, GM maps were added as covariates (nuisance variables) in the RSN fMRI analysis model (GLM). The BOLD-related group differences in the lateral visual and auditory networks (reported above) did not survive correction for GM. One possible explanation for this is that GM differences account for a significant part of the group differences in functional RSN data. Another possibility is that as adding GM as a covariate changes the degrees of freedom in the model, such that the results no longer reach significance. No significant correlations were found between the percentage signal change in the functional data and the GM values for the same areas. There was also virtually no overlap between those regions found to be significant in the RSN analysis and those significantly with reduced GM (Figure 5). This suggests that the group differences in RSN coherence are genuine and not the result of GM differences.

\section{DISCUSSION}

The results of this study suggest that regions associated with interoceptive and somatosensory functioning, including the processing of body image information, show hypoconnectivity in individuals with a history of $\mathrm{AN}$ (both current and past). As the recovered AN group were not currently malnourished, the observed differences cannot be solely explained by starvationassociated brain atrophy. Rather, these results suggest that $\mathrm{AN}$ is associated with enduring functional differences in brain organization, which could be either a scar of the illness or a premorbid feature, which represents an illness-related biomarker. In contrast to our previous resting-state study in recovered AN (19), we did not observe any differences in the DMN network. This could be due to methodological differences, as we previously measured resting-state neural activity after a symptom-provocation paradigm, which may have primed disorder-related preoccupation and ruminations associated with DMN activity.

The lateral visual network was described by Beckman and colleagues (43) as one which includes lateral visual cortical areas: the occipital pole, occipito-temporal junction, and superior parietal regions. This network has been implicated visual or visuo-spatial attention (50). We found, decreased connectivity in this network within the right temporal/temporal-occipital fusiform cortex, an area associated with face and body recognition (51). The fusiform gyrus is shown to activate in response to body images in both healthy individuals and in $\operatorname{EDs}(52,53)$; however, a decreased response in this region has been found in those with AN (52). Decreased connectivity between the fusiform body area (the posterior region of the fusiform gyrus) and extrastriate body has previously been reported in $\mathrm{AN}$ and correlated with body image distortion (54). Consistent with our findings, Favaro and colleagues also reported decreased connectivity in both acute and recovered AN within the visual network, in a region also associated with visual perception of the body (30). In a study using a network-based statistical approach, Ehrlich and colleagues found decreased connectivity in a thalamo-insular subnetwork including the fusiform gyrus, which might explain the disturbance in homeostatis and perceived body state in AN (55). Our results, together with previous literature, suggest that brain circuits associated with body image processing are dysfunctional in AN, and may underpin features such as distorted body image perception and abnormal experience of the body, characteristic of AN.

The auditory network includes the primary and secondary auditory cortices, insular cortex, the anterior cingulate cortex, anterior supramarginal gyrus, and thalamus (43). There have been few papers which have examined the role of auditory resting state network and these have focused on its role in tinnitus (56, 57). There have, to date been none reporting any changes in its connectivity in AN. In this study, within the auditory network, decreased connectivity was found in a number of regions including the precentral and postcentral gyri. The precentral and postcentral gyri correspond to the primary motor and somatosensory regions, respectively, and interact to contribute to complex motor and somatosensory functions $(58,59)$. Dysfunction of both these regions have been reported in $\mathrm{AN}$ in response to viewing other women's bodies (60), and increased activity within the precentral gyrus is seen in AN in response to viewing happy facial expressions (61). In addition, reduced connectivity of the postcentral gyrus with the sensorimotor network has previously been 

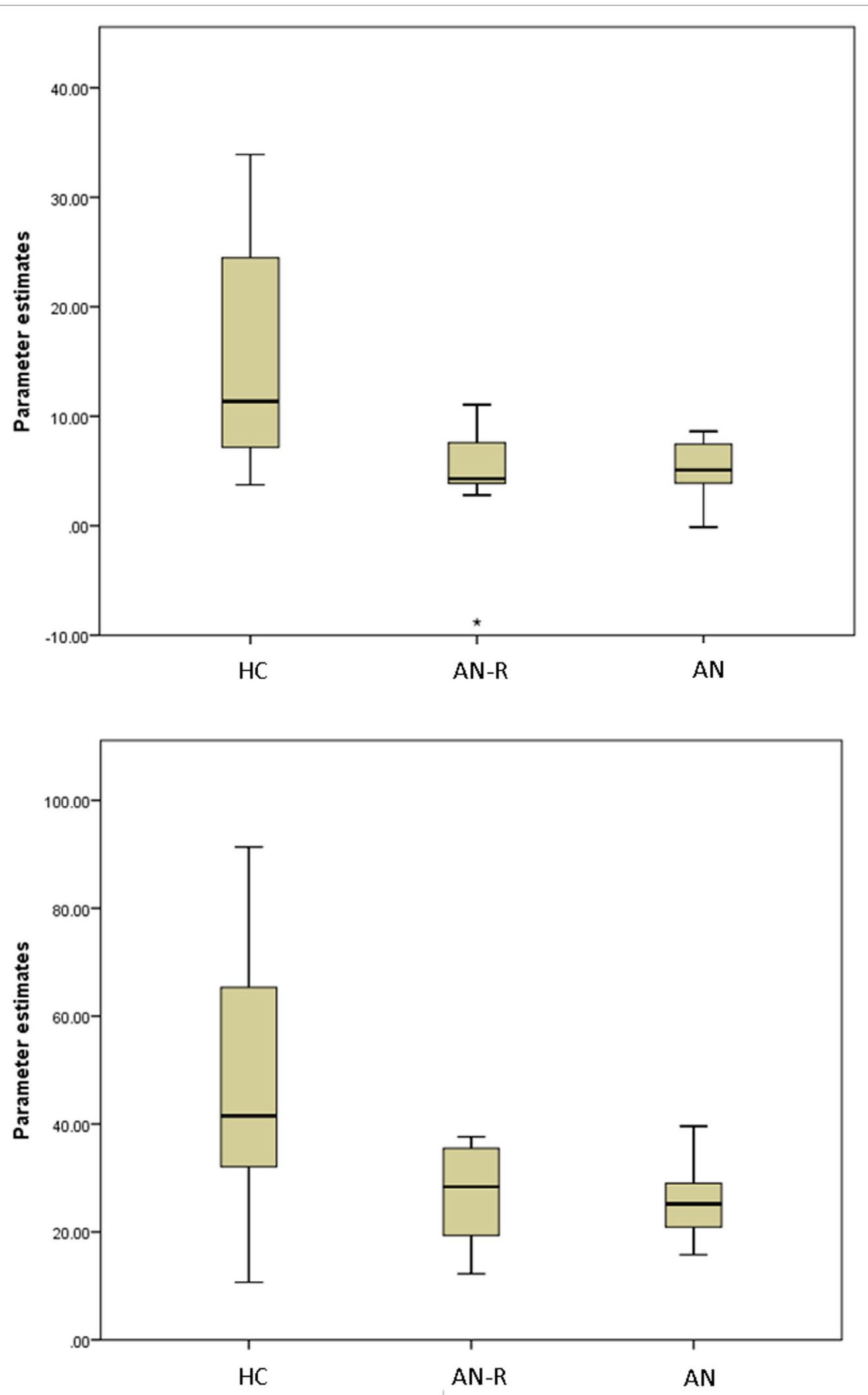

FIGURE 4 | Box plots of extracted BETA values in the HC, AN-R and AN groups in the Lateral Visual Network (Top) and Auditory Network (Bottom). Asterisk represents an outlier in the group. 
TABLE 2 | Global gray matter (GM), white matter (WM) and cerebrospinal fluid (CSF) volumes expressed as a percentage of total brain volume in the three groups ( $M \pm S D$, one-way ANOVA $p$-scores).

\begin{tabular}{lcccl}
\hline & HC & AN-R & AN & p-Score \\
& $\mathbf{N ( 1 6 )}$ & $\mathbf{N ( 1 4 )}$ & $\mathbf{N ( 1 2 )}$ & \\
\cline { 2 - 5 } WM volume & $37.5 \pm 1.4$ & $37.8 \pm 1.1$ & $37.1 \pm 1.3$ & 0.45 \\
GM volume & $43.0 \pm 1.1$ & $42.2 \pm 1.6$ & $40.0 \pm 4.5$ & 0.013 \\
CSF & $19.5 \pm 1.6$ & $20.0 \pm 2.2$ & $22.2 \pm 2.3$ & 0.004
\end{tabular}

reported in $\mathrm{AN}$ compared to $\mathrm{HC}$, suggested to reflect reduced somatosensory responsiveness in $\mathrm{AN}$ (62). In the McFadden study, reduction of postcentral gyrus activation in AN-R group compared to $\mathrm{HC}$ failed to reach significance, but a reduction in the postcentral gyrus in the AN group compared to the AN-R group was found-a difference which we did not see.

Decreased connectivity of the insula within the auditory network was also observed. Dysfunction of the insula has
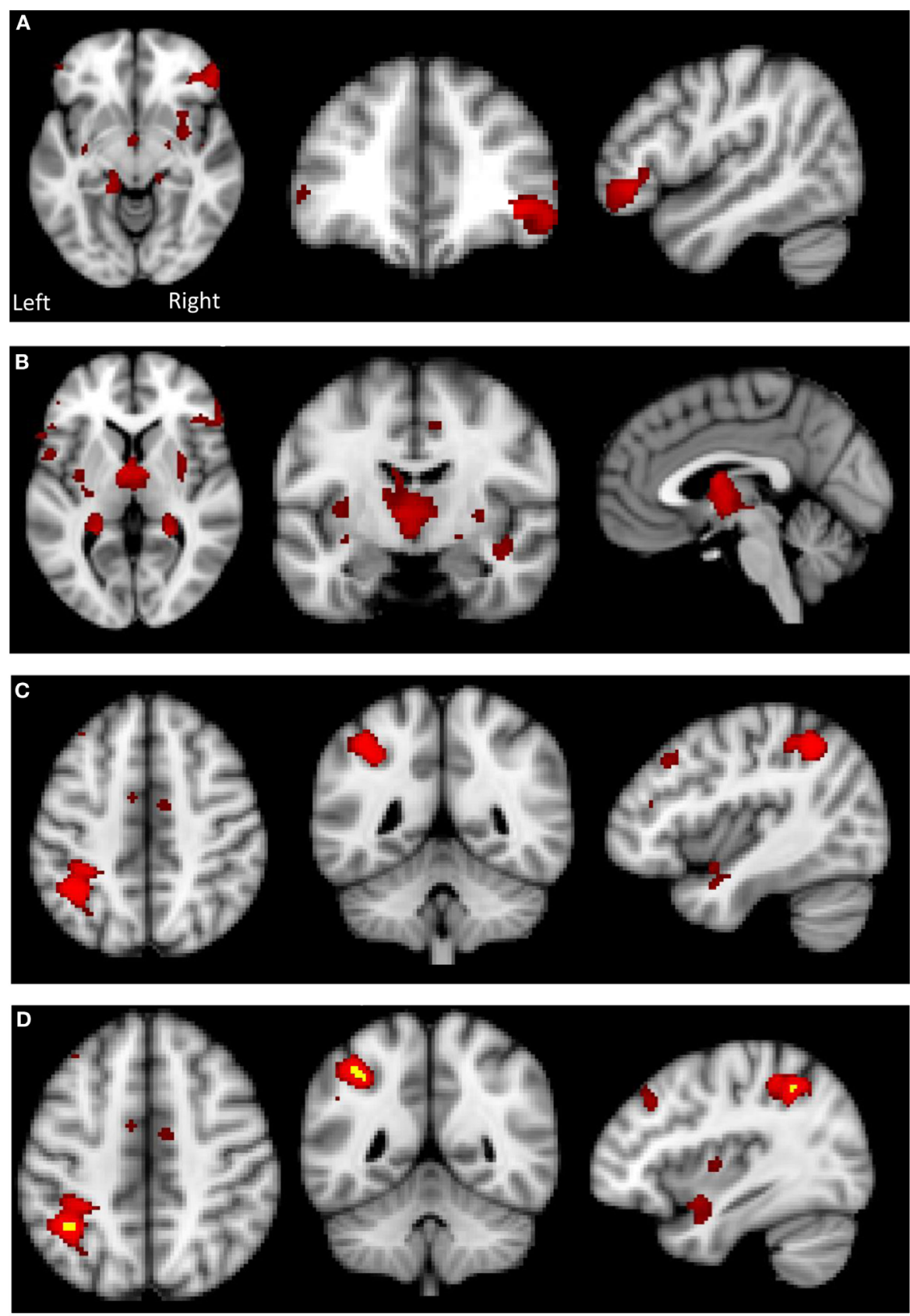

FIGURE 5 | Top: gray matter (GM) reduction in the anorexia nervosa (AN) group compared to HC in (A) the lateral prefrontal cortex, (B) putamen and thalamus, (C) left supramarginal gyrus/superior parietal lobule. Bottom: (D) overlap in GM reduction in the AN group (red) and the AN-R group (yellow) in the left supramarginal gyrus/superior parietal lobule. 
often been reported in neuroimaging studies of AN (63-66). The insula is a region of the brain with multiple complex functions, and is extensively connected to many regions in the brain, making it central to neural communication $(67,68)$. There is consistent evidence of interoceptive deficits and insula dysfunction in AN (12). The insula is implicated in the regulation of appetite (69), processing of taste information (70), and sensitivity to pain (71), all of which are shown to be aberrant in AN (72-74). Interestingly, in the context of the other regions of altered functional connectivity reported in this study, the insula has been associated with interoceptive processing, and providing a sense of the physiological condition of the body (75), the dysfunction of which could underpin distorted body image perception in AN (68).

We performed a VBM on the structural data, a technique which allows investigation of local concentrations of brain tissue, through voxel-wise comparison of multiple brain images. This approach requires no a priori hypotheses about regions of possible differences between groups, and it allows comprehensive measurements throughout the entire brain.

We found reduced GM in parts of the postcentral gyrus and insula cortex in the AN group. Reductions in these regions were no longer seen in the AN-R group, while a reduction in the supramarginal/angular gyrus region was shown in both AN groups. This raises the possibility that although there may be structural recovery from atrophy caused by malnourishment in regions responsible for interoceptive awareness of the bodily state and body image perception, functional changes may have taken place, which do not recover. Indeed, deficits in interoceptive processes and body image perception are also found in individuals recovered from $\mathrm{AN}(76,77)$. In line with our results, investigations of GM differences in AN have often reported widespread reductions. A recent systematic review reported reductions in the insula, frontal operculum, occipital, medial temporal, and cingulate cortex, but emphasized the inconsistency of results across samples and methodologies (78). In line with our finding of global GM loss in the AN but not the AN-R group, brain volume has also been shown to increase following weight gain and recovery (79-81), further supporting the possible role of starvation-related brain atrophy in the psychopathology of AN.

This study has several methodological limitations of note, as well as some important strengths. While our participants were asked to fast overnight prior to the resting-state scan to control for levels of hunger, ED research has been criticized for failing to control for the effects of dehydration and starvation, particularly in relation to brain matter volume (82). The effects of this were shown in a study by Frank and colleagues (83), in which GM volume was investigated in a sample of individuals with AN who had been on a strict meal plan for 1-2 weeks, in order to avoid the effects of malnutrition. Controlling for age, comorbid diagnoses, and brain volume, the authors found increased volume in the orbitofrontal cortex and right insula in AN (current and recovered) compared to controls (83). These results emphasize
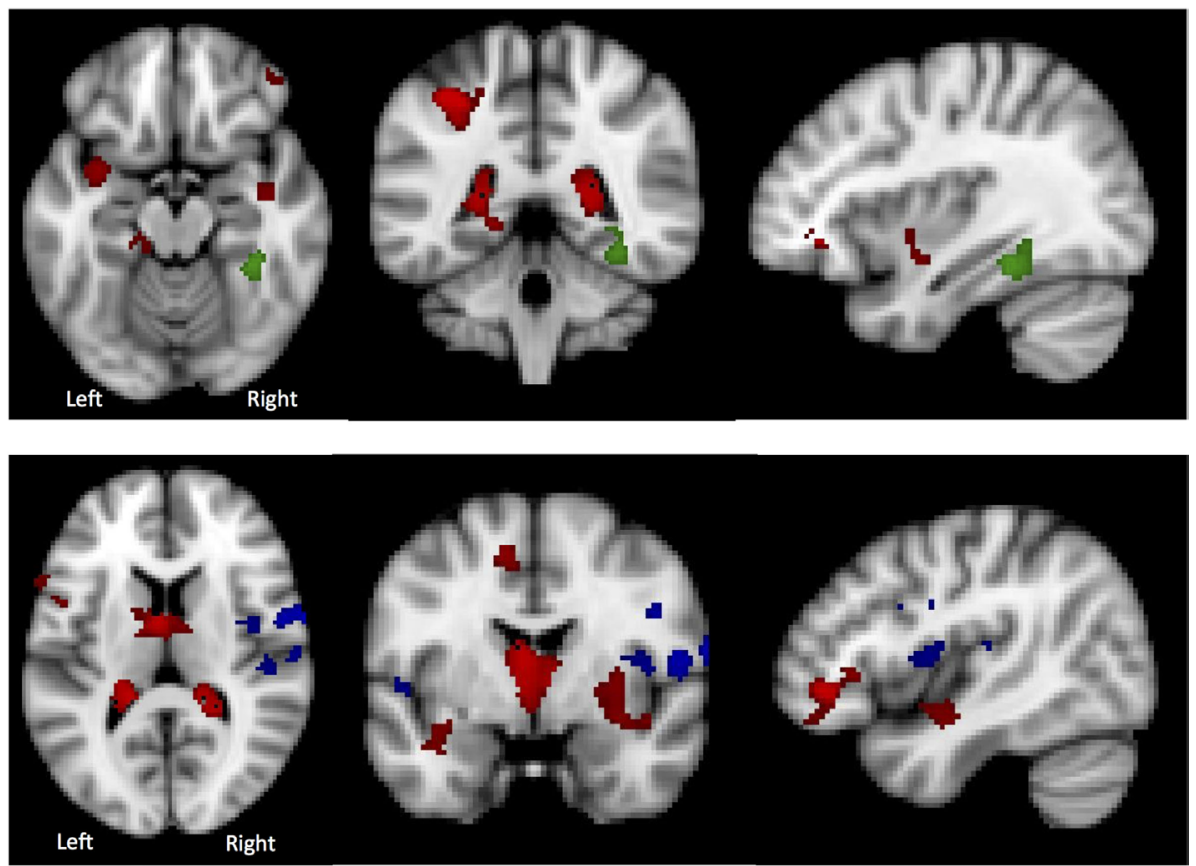

FIGURE 6 | Top: in the Lateral Visual Network (Green), the region in which there is a group difference is distinct from those regions in which there are significant gray matter (GM) differences (HC > AN) (shown in red). Bottom: in the Auditory Network (blue), the region in which there is a group difference is distinct from those regions in which there are significant GM differences (HC > AN) (shown in red). 
the importance of controlling for malnutrition and global brain volume in future AN research.

As with many studies in this area, the sample size used was fairly small. However, we did isolate a sample of purely restrictive AN. A number of resting-state studies in AN have included both restrictive and binge/purge AN subtypes and, as such, are unable to control for the possibility of within-group variability in brain network activity, which may be related to important differences in psychopathology between subtypes of AN. However, future research may benefit from larger sample sizes enabling the inclusion and comparison of both AN subtypes. In addition, a number of participants had comorbid anxiety or depression diagnoses and, as such, were taking serotonergic antidepressants and/or antipsychotic medications. Antidepressant and antipsychotic medications have been shown to alter resting neural connectivity (84-86), and so the effects of these medications, or indeed comorbid diagnoses, on our results cannot be ruled out.

\section{CONCLUSION}

Consistent with our predictions and with clinical characteristics of $\mathrm{AN}$ we have demonstrated decreased connectivity in regions associated with somatosensory and interoceptive function, as well as body image processing in individuals with current $\mathrm{AN}$ and those recovered from AN. These results are consistent with clinical and research findings of deficits

\section{REFERENCES}

1. Berkman ND, Lohr KN, Bulik CM. Outcomes of eating disorders: a systematic review of the literature. Int J Eat Disord (2007) 40:293-309. doi:10.1002/ eat.20369

2. Arcelus J, Mitchell AJ, Wales J, Nielsen S. Mortality rates in patients with anorexia nervosa and other eating disorders. A meta-analysis of 36 studies. Arch Gen Psychiatry (2011) 68:724-31. doi:10.1001/archgenpsychiatry. 2011.74

3. Mathers CD, Vos ET, Stevenson CE, Begg SJ. The Australian Burden of Disease Study: measuring the loss of health from diseases, injuries and risk factors. Med J Aust (2000) 172:592-6.

4. Bulik CM. The challenges of treating anorexia nervosa. Lancet (2014) 383:105-6. doi:10.1016/S0140-6736(13)61940-6

5. National Institute for Health and Care Excellence. Eating Disorders. Core Interventions in the Treatment and Management of Anorexia Nervosa, Bulimia Nervosa and Related Eating Disorders. CG09. London: National Institute for Health and Care Excellence (2004).

6. McKnight RF, Park RJ. Atypical antipsychotics and anorexia nervosa: a review. Eur Eat Disord Rev (2010) 18:10-21. doi:10.1002/erv.988

7. Watson H, Bulik C. Update on the treatment of anorexia nervosa: review of clinical trials, practice guidelines and emerging interventions. Psychol Med (2012) 10:1-24. doi:10.1017/S0033291712002620

8. Balestrieri M, Oriani MG, Simoncini A, Bellantuono C. Psychotropic drug treatment in anorexia nervosa. Search for differences in efficacy/tolerability between adolescent and mixed-age population. Eur Eat Disord Rev (2013) 21:361-73. doi:10.1002/erv.2240

9. Park RJ, Dunn BD, Barnard PJ. Schematic models and modes of mind in anorexia nervosa I: a novel process account. Int J Cogn Ther (2011) 4:415-37. doi:10.1521/ijct.2011.4.4.415

10. Park RJ, Dunn BD, Barnard PJ. Schematic models and modes of mind in anorexia nervosa II: implications for treatment and course. Int J Cogn Ther (2012) 5:86-98. doi:10.1521/ijct.2012.5.1.86 in interoceptive awareness in AN, abnormalities in the way the body is experienced, and body image distortion. Despite normal global GM volumes in recovered AN individuals, whose weight is fully restored, functional changes in RSNs remain. These may contribute to more persistent body image issues and potentially represent a vulnerability to relapse. Future research would benefit from larger sample sizes allowing the comparison of AN subtypes, and stricter controls on nutrition and hydration prior to imaging.

\section{ETHICS STATEMENT}

This study was carried out in accordance with the recommendations of NRES South Central-Oxford A Research Ethics Committee. The protocol was approved by the NRES South Central-Oxford A Research Ethics Committee.

\section{AUTHOR CONTRIBUTIONS}

JS, LG, CH, and RP designed research; JS, LG, and NF acquired and analyzed the data; JS, LG, CH, NF, and RP wrote the manuscript.

\section{FUNDING}

This research was funded by RP's Medical Research Council Confidence in Concept Award, number MC_PC_12020 and HEFCE Clinical Senior Lecturer Awards.

11. Kaye WH, Fudge JL, Paulus M. New insights into symptoms and neurocircuit function of anorexia nervosa. Nat Rev Neurosci (2009) 10:573-84. doi:10.1038/nrn2682

12. Kaye WH, Wierenga CE, Bailer UF, Simmons AN, Wagner A, BischoffGrethe A. Does a shared neurobiology for foods and drugs of abuse contribute to extremes of food ingestion in anorexia and bulimia nervosa? Biol Psychiatry (2013) 73:836-42. doi:10.1016/j.biopsych.2013. 01.002

13. Brookes MJ, Woolrich M, Luckhoo H, Price D, Hale JR, Stephenson MC, et al. Investigating the electrophysiological basis of resting state networks using magnetoencephalography. Proc Natl Acad Sci U S A (2011) 108:16783-8. doi:10.1073/pnas.1112685108

14. Biswal B, Yetkin FZ, Haughton VM, Hyde JS. Functional connectivity in the motor cortex of resting human brain using echo-planar MRI. Magn Reson Med (1995) 34:537-41.

15. van den Heuvel MP, Hulshoff Pol HE. Exploring the brain network: a review on resting-state fMRI functional connectivity. Eur Neuropsychopharmacol (2010) 20:519-34. doi:10.1016/j.euroneuro.2010.03.008

16. Beckmann CF, Smith SM. Probabilistic independent component analysis for functional magnetic resonance imaging. IEEE Trans Med Imaging (2004) 23:137-52. doi:10.1109/TMI.2003.822821

17. Cole DM, Smith SM, Beckmann CF. Advances and pitfalls in the analysis and interpretation of resting-state fMRI data. Front Syst Neurosci (2010) 4:8. doi:10.3389/fnsys. 2010.00008

18. Gaudio S, Wiemerslage L, Brooks SJ, Schioth HB. A systematic review of resting-state functional-MRI studies in anorexia nervosa: evidence for functional connectivity impairment in cognitive control and visuospatial and body-signal integration. Neurosci Biobehav Rev (2016) 71:578-89. doi:10.1016/j.neubiorev.2016.09.032

19. Cowdrey FA, Filippini N, Park RJ, Smith SM, McCabe C. Increased resting state functional connectivity in the default mode network in recovered anorexia nervosa. Hum Brain Mapp (2012) 35:483-91. doi:10.1002/ hbm.22202 
20. Raichle ME, MacLeod AM, Snyder AZ, Powers WJ, Gusnard DA, Shulman GL. A default mode of brain function. Proc Natl Acad Sci U S A (2001) 98:676-82. doi:10.1073/pnas.98.2.676

21. Cowdrey FA, Park RJ, Harmer CJ, McCabe C. Increased neural processing of rewarding and aversive food stimuli in recovered anorexia nervosa. Biol Psychiatry (2011) 70:736-43. doi:10.1016/j.biopsych.2011.05.028

22. Bischoff-Grethe A, McCurdy D, Grenesko-Stevens E, Irvine LE, Wagner A, Yau WY, et al. Altered brain response to reward and punishment in adolescents with anorexia nervosa. Psychiatry Res (2013) 214:331-40. doi:10.1016/j.pscychresns.2013.07.004

23. Boehm I, Geisler D, King JA, Ritschel F, Seidel M, Deza Araujo Y, et al. Increased resting state functional connectivity in the fronto-parietal and default mode network in anorexia nervosa. Front Behav Neurosci (2014) 8:346. doi:10.3389/fnbeh.2014.00346

24. Boehm I, Geisler D, Tam F, King JA, Ritschel F, Seidel M, et al. Partially restored resting-state functional connectivity in women recovered from anorexia nervosa. J Psychiatry Neurosci (2016) 41:377-85. doi:10.1503/ jpn.150259

25. Favaro A, Clementi M, Manara R, Bosello R, Forzan M, Bruson A, et al. Catechol-O-methyltransferase genotype modifies executive functioning and prefrontal functional connectivity in women with anorexia nervosa. J Psychiatry Neurosci (2012) 37:120068. doi:10.1503/jpn.120068

26. Kullmann S, Giel KE, Teufel M, Thiel A, Zipfel S, Preissl H. Aberrant network integrity of the inferior frontal cortex in women with anorexia nervosa. Neuroimage Clin (2014) 4:615-22. doi:10.1016/j.nicl.2014.04.002

27. Collantoni E, Michelon S, Tenconi E, Degortes D, Titton F, Manara R, et al. Functional connectivity correlates of response inhibition impairment in anorexia nervosa. Psychiatry Res (2016) 247:9-16. doi:10.1016/j. pscychresns.2015.11.008

28. Biezonski D, Cha J, Steinglass J, Posner J. Evidence for thalamocortical circuit abnormalities and associated cognitive dysfunctions in underweight individuals with anorexia nervosa. Neuropsychopharmacology (2016) 41:1560-8. doi:10.1038/npp.2015.314

29. Gaudio S, Piervincenzi C, Beomonte Zobel B, Romana Montecchi F, Riva G, Carducci F, et al. Altered resting state functional connectivity of anterior cingulate cortex in drug naive adolescents at the earliest stages of anorexia nervosa. Sci Rep (2015) 5:10818. doi:10.1038/srep10818

30. Favaro A, Santonastaso P, Manara R, Bosello R, Bommarito G, Tenconi E, et al. Disruption of visuospatial and somatosensory functional connectivity in anorexia nervosa. Biol Psychiatry (2012) 72(10):864-70. doi:10.1016/j. biopsych.2012.04.025

31. Phillipou A, Abel LA, Castle DJ, Hughes ME, Nibbs RG, Gurvich C, et al. Resting state functional connectivity in anorexia nervosa. Psychiatry Res (2016) 251:45-52. doi:10.1016/j.pscychresns.2016.04.008

32. Amianto F, D’Agata F, Lavagnino L, Caroppo P, Abbate-Daga G, Righi D, et al. Intrinsic connectivity networks within cerebellum and beyond in eating disorders. Cerebellum (2013) 12:623-31. doi:10.1007/s12311-013-0471-1

33. Geisler D, Borchardt V, Lord AR, Boehm I, Ritschel F, Zwipp J, et al. Abnormal functional global and local brain connectivity in female patients with anorexia nervosa. J Psychiatry Neurosci (2016) 41:6-15. doi:10.1503/ jpn. 140310

34. Lord A, Ehrlich S, Borchardt V, Geisler D, Seidel M, Huber S, et al. Brain parcellation choice affects disease-related topology differences increasingly from global to local network levels. Psychiatry Res (2016) 249:12-9. doi:10.1016/j. pscychresns.2016.02.001

35. Brooks SJ, Rask-Andersen M, Benedict C, Schiöth HB. A debate on current eating disorder diagnoses in light of neurobiological findings: is it time for a spectrum model? BMC Psychiatry (2012) 12:76. doi:10.1186/1471-244X-12-76

36. American Psychiatric Association. Diagnostic and Statistical Manual of Mental Disorders. 5th ed. Washington, DC: American Psychiatric Association (2013). Available from: http://blog.apastyle.org/apastyle/2013/08/how-tocite-the-dsm5-in-apa-style.html

37. Beglin SJ, Fairburn CG. Eating Disorder Examination Questionnaire (6.0). New York: Guilford Press (2008).

38. Mazure CM, Halmi KA, Sunday SR, Romano SJ, Einhorn AM. The YaleBrown-Cornell Eating Disorder Scale: development, use, reliability and validity. J Psychiatr Res (1994) 28:425-45.

39. Beck AT, Steer RA, Brown GK. Manual for the Beck Depression Inventory-II. San Antonio, TX: Psychological Corporation (1996).
40. Spielberger CD. Manual for the State-Trait Anxiety Inventory (Form Y). Palo Alto, CA: Mind Garden (1983).

41. Nelson HE, Willison JR. National Adult Reading Test (NART): Test Manual. Windsor: NFER-Nelson (1991).

42. Scaife JC, Godier LR, Reinecke A, Harmer CJ, Park RJ. Differential activation of the frontal pole to high vs low calorie foods: the neural basis of food preference in anorexia nervosa? Psychiatry Res (2016) 258:44-53. doi:10.1016/j. pscychresns.2016.10.004

43. Beckmann CF, DeLuca M, Devlin JT, Smith SM. Investigations into resting-state connectivity using independent component analysis. Philos Trans $R$ Soc Lond B Biol Sci (2005) 360:1001-13. doi:10.1098/rstb.2005.1634

44. Filippini N, MacIntosh BJ, Hough MG, Goodwin GM, Frisoni GB, Smith SM, et al. Distinct patterns of brain activity in young carriers of the APOEepsilon4 allele. Proc Natl Acad Sci U S A (2009) 106:7209-14. doi:10.1073/ pnas. 0811879106

45. Nichols TE, Holmes AP. Nonparametric permutation tests for functional neuroimaging: a primer with examples. Hum Brain Mapp (2002) 15:1-25. doi:10.1002/hbm.1058

46. Hayasaka S, Phan KL, Liberzon I, Worsley KJ, Nichols TE. Nonstationary cluster-size inference with random field and permutation methods. Neuroimage (2004) 22:676-87. doi:10.1016/j.neuroimage.2004.01.041

47. Barnes A, Bullmore ET, Suckling J. Endogenous human brain dynamics recover slowly following cognitive effort. PLoS One (2009) 4:e6626. doi:10.1371/journal.pone.0006626

48. Douaud G, Smith S, Jenkinson M, Behrens T, Johansen-Berg H, Vickers $\mathrm{J}$, et al. Anatomically related grey and white matter abnormalities in adolescent-onset schizophrenia. Brain (2007) 130:2375-86. doi:10.1093/brain/ awm 184

49. Oakes TR, Fox AS, Johnstone T, Chung MK, Kalin N, Davidson RJ. Integrating VBM into the general linear model with voxelwise anatomical covariates. Neuroimage (2007) 34:500-8. doi:10.1016/j.neuroimage.2006.10.007

50. Yang YL, Deng HX, Xing GY, Xia XL, Li HF. Brain functional network connectivity based on a visual task: visual information processing-related brain regions are significantly activated in the task state. Neural Regen Res (2015) 10:298-307. doi:10.4103/1673-5374.152386

51. Peelen MV, Downing PE. Selectivity for the human body in the fusiform gyrus. J Neurophysiol (2005) 93:603-8. doi:10.1152/jn.00513.2004

52. Uher R, Murphy T, Friederich HC, Dalgleish T, Brammer MJ, Giampietro $\mathrm{V}$, et al. Functional neuroanatomy of body shape perception in healthy and eating-disordered women. Biol Psychiatry (2005) 58:990-7. doi:10.1016/j. biopsych.2005.06.001

53. Wagner A, Ruf M, Braus DF, Schmidt MH. Neuronal activity changes and body image distortion in anorexia nervosa. Neuroreport (2003) 14:2193-7. doi:10.1097/01.wnr.0000089567.45990.d9

54. Suchan B, Bauser DS, Busch M, Schulte D, Grönemeyer D, Herpertz S, et al. Reduced connectivity between the left fusiform body area and the extrastriate body area in anorexia nervosa is associated with body image distortion. Behav Brain Res (2012) 241:80-5. doi:10.1016/j.bbr.2012. 12.002

55. Ehrlich S, Lord AR, Geisler D, Borchardt V, Boehm I, Seidel M, et al. Reduced functional connectivity in the thalamo-insular subnetwork in patients with acute anorexia nervosa. Hum Brain Mapp (2015) 36:1772-81. doi:10.1002/ hbm. 22736

56. Maudoux A, Lefebvre P, Cabay JE, Demertzi A, Vanhaudenhuyse A, Laureys S, et al. Auditory resting-state network connectivity in tinnitus: a functional MRI study. PLoS One (2012) 7:e36222. doi:10.1371/journal.pone. 0036222

57. Maudoux A, Lefebvre P, Cabay JE, Demertzi A, Vanhaudenhuyse A, Laureys $\mathrm{S}$, et al. Connectivity graph analysis of the auditory resting state network in tinnitus. Brain Res (2012) 1485:10-21. doi:10.1016/j.brainres.2012.05.006

58. Sun H, Blakely TM, Darvas F, Wander JD, Johnson LA, Su DK, et al. Sequential activation of premotor, primary somatosensory and primary motor areas in humans during cued finger movements. Clin Neurophysiol (2015) 7(5):e36222. doi:10.1016/j.clinph.2015.01.005

59. Rao SM, Binder JR, Bandettini PA, Hammeke TA, Yetkin FZ, Jesmanowicz A, et al. Functional magnetic resonance imaging of complex human movements. Neurology (1993) 43:2311-8.

60. Vocks S, Busch M, Gronemeyer D, Schulte D, Herpertz S, Suchan B. Neural correlates of viewing photographs of one's own body and another woman's 
body in anorexia and bulimia nervosa: an fMRI study. J Psychiatry Neurosci (2010) 35:163-76. doi:10.1503/jpn.090048

61. Fonville L, Giampietro V, Surguladze S, Williams S, Tchanturia K. Increased BOLD signal in the fusiform gyrus during implicit emotion processing in anorexia nervosa. Neuroimage Clin (2014) 4:266-73. doi:10.1016/j. nicl.2013.12.002

62. McFadden KL, Tregellas JR, Shott ME, Frank GK. Reduced salience and default mode network activity in women with anorexia nervosa. J Psychiatry Neurosci (2014) 39:178-88. doi:10.1503/jpn.130046

63. Kojima S, Nagai N, Nakabeppu Y, Muranaga T, Deguchi D, Nakajo M, et al. Comparison of regional cerebral blood flow in patients with anorexia nervosa before and after weight gain. Psychiatry Res (2005) 140:251-8. doi:10.1016/j. pscychresns.2005.08.002

64. Sachdev P, Mondraty N, Wen W, Gulliford K. Brains of anorexia nervosa patients process self-images differently from non-self-images: an fMRI study. Neuropsychologia (2008) 46:2161-8. doi:10.1016/j.neuropsychologia. 2008.02.031

65. Wagner A, Aizenstein H, Mazurkewicz L, Fudge J, Frank GK, Putnam $\mathrm{K}$, et al. Altered insula response to taste stimuli in individuals recovered from restricting-type anorexia nervosa. Neuropsychopharmacology (2008) 33:513-23. doi:10.1038/sj.npp.1301443

66. Redgrave GW, Bakker A, Bello NT, Caffo BS, Coughlin JW, Guarda AS, et al. Differential brain activation in anorexia nervosa to fat and thin words during a Stroop task. Neuroreport (2008) 19:1181-5. doi:10.1097/ WNR.0b013e32830a70f2

67. Nunn K, Frampton I, Gordon I, Lask B. The fault is not in her parents but in her insula - a neurobiological hypothesis of anorexia nervosa. Eur Eat Disord Rev (2008) 16:355-60. doi:10.1002/erv.890

68. Nagai M, Kishi K, Kato S. Insular cortex and neuropsychiatric disorders: a review of recent literature. Eur Psychiatry (2007) 22:387-94. doi:10.1016/j. eurpsy.2007.02.006

69. Shelley BP, Trimble MR. The insular lobe of reil - its anatamico-functional, behavioural and neuropsychiatric attributes in humans - a review. World J Biol Psychiatry (2004) 5:176-200. doi:10.1080/15622970410029933

70. Pritchard TC, Macaluso DA, Eslinger PJ. Taste perception in patients with insular cortex lesions. Behav Neurosci (1999) 113:663-71.

71. Starr CJ, Sawaki L, Wittenberg GF, Burdette JH, Oshiro Y, Quevedo AS, et al. Roles of the insular cortex in the modulation of pain: insights from brain lesions. J Neurosci (2009) 29:2684-94. doi:10.1523/JNEUROSCI.5173-08.2009

72. Aschenbrenner K, Scholze N, Joraschky P, Hummel T. Gustatory and olfactory sensitivity in patients with anorexia and bulimia in the course of treatment. J Psychiatr Res (2008) 43:129-37. doi:10.1016/j.jpsychires.2008.03.003

73. Garfinkel PE. Perception of hunger and satiety in anorexia nervosa. Psychol Med (1974) 4:309-15.

74. Bar KJ, Boettger S, Wagner G, Wilsdorf C, Gerhard UJ, Boettger MK, et al. Changes of pain perception, autonomic function, and endocrine parameters during treatment of anorectic adolescents. J Am Acad Child Adolesc Psychiatry (2006) 45:1068-76. doi:10.1097/01.chi.0000227876. 19909.48

75. Craig AD. How do you feel? Interoception: the sense of the physiological condition of the body. Nat Rev Neurosci (2002) 3:655-66. doi:10.1038/nrn894
76. Strigo IA, Matthews SC, Simmons AN, Oberndorfer T, Klabunde M, Reinhardt LE, et al. Altered insula activation during pain anticipation in individuals recovered from anorexia nervosa: evidence of interoceptive dysregulation. Int J Eat Disord (2013) 46:23-33. doi:10.1002/eat.22045

77. Lautenbacher S, Kraehe N, Krieg JC. Perception of body size and body satisfaction in recovered anorexic women: comparison with restrained and unrestrained eaters. Percept Mot Skills (1997) 84:1331-42.

78. Van den Eynde F, Suda M, Broadbent H, Guillaume S, Van den Eynde M, Steiger $\mathrm{H}$, et al. Structural magnetic resonance imaging in eating disorders: a systematic review of voxel-based morphometry studies. Eur Eat Disord Rev (2012) 20:94-105. doi:10.1002/erv.1163

79. Roberto CA, Mayer LE, Brickman AM, Barnes A, Muraskin J, Yeung LK, et al. Brain tissue volume changes following weight gain in adults with anorexia nervosa. Int J Eat Disord (2011) 44:406-11. doi:10.1002/eat.20840

80. Wagner A, Greer P, Bailer UF, Frank GK, Henry SE, Putnam K, et al. Normal brain tissue volumes after long-term recovery in anorexia and bulimia nervosa. Biol Psychiatry (2006) 59:291-3. doi:10.1016/j.biopsych.2005.06.014

81. Lazaro L, Andres S, Calvo A, Cullell C, Moreno E, Plana MT, et al. Normal gray and white matter volume after weight restoration in adolescents with anorexia nervosa. Int J Eat Disord (2013) 46:841-8. doi:10.1002/eat.22161

82. Frank GK. Advances from neuroimaging studies in eating disorders. CNS Spectr (2015) 20(4):391-400. doi:10.1017/S1092852915000012

83. Frank GK, Shott ME, Hagman JO, Mittal VA. Alterations in brain structures related to taste reward circuitry in ill and recovered anorexia nervosa and in bulimia nervosa. Am J Psychiatry (2013) 2013:1152-60. doi:10.1176/appi. ajp.2013.12101294

84. van de Ven V, Wingen M, Kuypers KPC, Ramaekers JG, Formisano E. Escitalopram decreases cross-regional functional connectivity within the default-mode network. PLoS One (2013) 8:e68355. doi:10.1371/journal. pone. 0068355

85. McCabe C, Mishor Z. Antidepressant medications reduce subcortical-cortical resting-state functional connectivity in healthy volunteers. Neuroimage (2011) 57:1317-23. doi:10.1016/j.neuroimage.2011.05.051

86. Lui S, Li T, Deng W, Jiang L, Wu Q, Tang H, et al. Short-term effects of antipsychotic treatment on cerebral function in drug-naive first-episode schizophrenia revealed by "resting state" functional magnetic resonance imaging. Arch Gen Psychiatry (2010) 67:783-92. doi:10.1001/archgenpsychiatry. 2010.84

Conflict of Interest Statement: $\mathrm{CH}$ has received consultancy fees from Lundbeck, P1vital, Astra Zeneca and Servier. She is a company director of Oxford Psychologists Ltd. and holds shares in the same company. $\mathrm{CH}$ has received grant funding from UCB, J\&J, Astra Zeneca, Lundbeck, and Sunovion. JS, LG, NF, and RP report no biomedical financial interests or potential conflicts of interest.

Copyright $\odot 2017$ Scaife, Godier, Filippini, Harmer and Park. This is an open-access article distributed under the terms of the Creative Commons Attribution License (CC BY). The use, distribution or reproduction in other forums is permitted, provided the original author(s) or licensor are credited and that the original publication in this journal is cited, in accordance with accepted academic practice. No use, distribution or reproduction is permitted which does not comply with these terms. 